\title{
Quantum of temperature necessary for the thermal electron excitation in a one-dimensional metal
}

\begin{abstract}
An analysis of the electron specific heat of a quasi one-dimensional free-electron system leads to conclusion that some minimal quantum of temperature should be applied to the system in order to promote the excitation of an electron present on the Fermi level. The value of the minimal excitation temperature depends on the length of the one-dimensional potential box containing the electrons, the quantum state of the electron, and the actual temperature of the system as well as the fundamental constants of nature.
\end{abstract}

Keywords: temperature, thermal electron excitation, one-dimensional metal

\author{
Volume 2 Issue I - 2018
}

Stanislaw Olszewski

Institute of Physical Chemistry, Polish Academy of Sciences, Poland

Correspondence: Stanislaw Olszewski, Institute of Physical Chemistry, Polish Academy of Sciences, Poland, Email olsz@ichf.edu.pl

Received: January 22, 2018| Published: February 07, 2018

\section{Introduction}

A one-dimensional metal is a well-known model applied both in quantum physics and chemistry; see e.g. ${ }^{1-4} \mathrm{We}$ assume that electrons are enclosed in a tube of a constant potential which is so thin that for low energies the excitations along the tube length can be only admitted. In this case the transversal excitations are of much higher energy than the longitudinal ones and are usually neglected.

The energies dependent on the box length $\mathrm{L}$ are

$$
E_{n}=\frac{n^{2} h^{2}}{8 m L^{2}}
$$

where $\mathrm{n}$ is the electron quantum number. The lowest excitation energy of $E_{n}$ is

$$
\Delta E_{n}=E_{n+1}-E_{n}=\frac{(n+1)^{2}-n^{2}}{8 m L^{2}} h^{2}=\frac{2 n+1}{8 m L^{2}} h^{2} \approx \frac{n h^{2}}{4 m L^{2}},
$$

where the last step in (2) holds for $n \gg 1$.

The electron specific heat of the electron ensemble is usually examined for a three-dimensional electron gas case. ${ }^{5-7}$ In such a study the contributions given by the individual electrons are regularly neglected and the quantum-statistical density of the whole electron ensemble is taken into account. However, because of the special boundary conditions applied to a one-dimensional metal, a different, i.e. partly non-statistical kind of approach, can be followed in calculating the electron specific heat done in the present paper.

\section{Electron specific heat calculated from a quantum oscillator}

Let us assume that electrons in the box are vibrating along the box length $L$ with the frequency $v$.

This frequency is easy to calculate for any quantum state $n$.

For a free electron moving with the velocity $v_{n}$ we have the relation

$$
m \frac{v_{n}^{2}}{2}=E_{n}
$$

where $E_{n}$ is taken from (1). This gives

$$
v_{n}=\left(\frac{2 E_{n}}{m}\right)^{1 / 2}=\left(\frac{n^{2} h^{2}}{4 m^{2} L^{2}}\right)^{1 / 2}=\frac{n h}{2 m L}
$$

In the next step the speed $v_{n}$ is coupled with the time period $t_{p n}$ necessary for an electron to travel from one of the box ends to another end, and back to the beginning electron position in the box. This velocity is represented by the formula

$$
v_{n}=\frac{2 L}{t_{p n}}
$$

An equality between (4) and (5) gives

$$
t_{p n}=\frac{4 m L^{2}}{n h},
$$

so the vibration frequency along $\mathrm{L}$ becomes

$$
v=\frac{1}{t_{p n}}=\frac{n h}{4 m L^{2}}
$$

A characteristic point is that the frequency in (7) can be referred to the energy difference in (2) by the formula

$$
\Delta E_{n}=h v=\frac{n h^{2}}{4 m L^{2}},
$$

on condition the quantum number $\mathrm{n}$ is large.

The frequency $v$ of the one-dimensional motion in (7) can be coupled with the temperature $\mathrm{T}$ by the well-known Planck's energy formula for the system of $\mathrm{N}$ one-dimensional oscillators ${ }^{8}$

$$
E=N \varepsilon_{0}+N E(T)
$$

where

$$
E(T)=\frac{N \mathrm{~h} v}{e^{h v / k T}-1}
$$

and $\varepsilon_{0}$ is a constant zero-point energy of the oscillator independent of T. The symbol $v$ is the frequency equal to (7), $\mathrm{k}$ is the Boltzmann 
constant. In Section 3 we calculate the specific heat of the electron oscillator on the basis of (9).

\section{Specific heat of the Planck's one-dimensional oscillator}

This kind of the electron specific heat can be obtained directly from the formula (10). We have

$$
c_{V}=\frac{1}{N} \frac{\partial E}{\partial T}=\frac{\partial}{\partial T}\left(\frac{h v}{e^{h v / k T}-1}\right)=-\frac{h v}{\left(e^{h v / k T}-1\right)^{2}} e^{h v / k T}\left(-\frac{1}{T^{2}}\right) \frac{h v}{k}=k\left(\frac{h v}{k T}\right)^{2} \frac{e^{h v / k T}}{\left(e^{h v / k T}-1\right)^{2}}
$$

The result in (11) is the well-known Einstein's formula for the specific heat of the vibrating atoms in a three-dimensional crystal, ${ }^{6,7,9}$ all atoms are having a definite frequency $v$. The only difference from the Einstein's result is dictated by the dimensionality of the oscillating system: the coefficient 3 before the formula in (11) is obtained in the Einstein case which corresponds to a three-dimensional oscillating object, for the one-dimensional electron oscillators considered in the present approach the number of 3 is replaced by 1 . Evidently the frequency $v$-entering the Planck's theory-is referred to the metal parameters by the formula (7).

\section{Balance of energy due to the temperature change with the excitation energy}

In order to promote an electron from the Fermi level, the excitation energy from that level represented by (2) and (8)-where $v$ is the frequency characteristic for the oscillations on the Fermi level-should be equal to the energy supplied by the temperature change $\Delta T$ :

$$
\Delta E=c_{V} \Delta T
$$

We obtain from (8) and (12) the result

$$
\frac{h v}{c_{V}}=\Delta T
$$

By dividing the both sides of (13) by $\mathrm{T}$ we obtain

$$
\frac{h v}{T c V}=\frac{h v}{k T} \frac{1}{x^{2}} \frac{\left(e^{x}-1\right)^{2}}{e^{x}}=\frac{1}{x} \frac{\left(e^{x}-1\right)^{2}}{e^{x}}=\frac{\Delta T}{T},
$$

on condition an abbreviated formula

$$
x=\frac{h v}{k T}
$$

is applied. Evidently we have $x$ tending to zero for very large T. This case is satisfied by equation (14).

A limiting value applicable for $\Delta T$ seems to be

$$
\Delta T \cong T,
$$

or

$$
\frac{\Delta T}{T} \cong 1,
$$

where $\mathrm{T}$ is the Planck's equilibrium temperature entering the energy (10) of the oscillator.

In this case also equation (14) is roughly satisfied on condition there is substituted the ratio (16a) and $x=1$. For we obtain from (14) the equation:

$$
\frac{\left(e^{x}-1\right)^{2}}{x e^{x}}=\frac{(e-1)^{2}}{e}=1
$$

which transformed into

$$
e^{2}-3 e+1=0
$$

gives

$$
e=\frac{3+\sqrt{9-4}}{2}=\frac{3+\sqrt{5}}{2}=1.5+1.118=2.618
$$

whereas the correct value of $e$ is 2.718

\section{Conclusion}

A one-dimensional non-interacting free electron system is studied in the case when the electron excitations are provided by an increase of the temperature. In the first step the electron particle behaviour is replaced by that of an oscillator. Next it is demonstrated that the specific heat of the oscillators is close to that given by the Einstein's formula for a metal, on condition the reduction of the metal dimensionality from 3 to 1 is taken into account In general it is shown that the quantum of temperature equal approximately to the equilibrium temperature of the Planck's oscillator is adequate to provide a thermal excitation of an electron to its next quantum level.

\section{Acknowledgment}

None.

\section{Conflict of interest}

None.

\section{References}

1. Eyring H, Walter J, Kimball GE. Quantum Chemistry. Wiley, USA, 1958.

2. Nikitine S, El-Komoss SG. Journal de chim Phys. 1954;51:129.

3. Olszewski S. Zeitschrift fuer Physik B. Condensed Matter. 1982;45:297.

4. Olszewski S, Rolinski T. Journal of Modern Physics. 2010;1:328.

5. Sommerfeld, Bethe H. Handbuch der Physik. In: Geiger H, Scheel K, editors. Germany, Springer; 1933.

6. Mott NF, Jones H. The Theory of the Properties of Metals and Alloys. USA, University Press, Oxford; 1958.

7. Ziman JM. Principles of the Theory of Solids. 2nd ed. Cambridge, University Press; 1972.

8. Planck M. Einführung in die Theorie der Wärme. 2nd ed. Germany, Hirzel S; 1932.

9. Einstein A. Annalen der Physik, 1907; 22: 180 and 800. 